\section{Breathing exercises for asthma: panacea or placebo?}

Mike G Pearson

\section{The finding that patients with asthma feel better after breathing exercises cannot be ignored}

B reathing is easy-we all do it all of the time from the moment we are born. So why should it be necessary to teach people how to breathe? How is it possible to get something wrong when it appears so simple? And, if it is wrong, can it be corrected?

In this issue of Thorax Holloway and West ${ }^{1}$ report a randomised trial of adding the Papworth technique-a combination of breathing and relaxation exercises first introduced for hyperventilation syndromes-to usual care in patients with asthma (see $p$ 1039). They recruited 85 volunteers with asthma from a primary care asthma register, 72 of whom completed the parallel group study and were followed up for 12 months. The group had relatively mild asthma with mean forced expiratory volume in $1 \mathrm{~s}\left(\mathrm{FEV}_{1}\right)$ $>90 \%$ predicted and thus, unsurprisingly, there were no significant improvements in the conventional physiological measures. However, the actively managed group who received five sessions of the Papworth technique, each lasting $1 \mathrm{~h}$, had significant improvements in the St George's Respiratory Questionnaire (SGRQ), symptoms and anxiety and depression scores.

This study raises as many questions as it answers. Why might breathing be amenable to change? Most homeostatic mechanisms (such as body temperature, hormonal cycles, blood sugar levels) are automatic and impervious to voluntary control. But breathing is different. The body's automatic "settings" can be overridden by voluntary control. It is possible to breath hold and, with training, to do so for prolonged periods (eg, several minutes as exemplified by the extreme diving sport). It is equally possible and easier to hyperventilate, so inducing hypocapnia and alkalosis. Alkalosis alters the levels of ionised calcium, nerve conduction speeds and smooth muscle control, especially of the vessels supplying the brain and heart. Cerebral blood flow may be reduced $40 \%$ by hyperventilation. Patients become aware of a myriad of symptoms that can include faintness, dizziness, chest tightness/discomfort and paraesthesia, especially of the hands. Some patients appear to hyperventilate on a regular basis leading to the description of "hyperventilation syndromes", ${ }^{2}$ although there is continuing controversy about their existence and some prefer terms such as "behavioural breathlessness". ${ }^{3}$

Lum in the 1960s recognised patients who had continuing symptoms despite normal cardiorespiratory physiology on standard testing and who appeared to be hyperventilating often with an irregular pattern of breathing. ${ }^{4}$ A programme of breathing and relaxation exercises was developed at Papworth Hospital and Lum reported observational data claiming that the Papworth technique could resolve symptoms in $70 \%$ or more of those he had defined as hyperventilating. His work-which included patients with asthma - was not well received by mainstream respiratory medicine.

There were two major reasons for the doubt at the time: (1) a prevalent belief that the hyperventilation syndromes were more likely to be psychological than physiological which, coupled to less than exacting definitions of diagnostic criteria and treatment evaluation, was understandable; and (2) there was an asthma treatment revolution that was backed by solid science. The new inhaled drugs were able to improve dramatically the lives of many patients with asthma, and the inhalers also addressed the airway inflammation. The mantra for managing asthma in the 1980s became "asthma is an inflammatory condition-prescribe an inhaled steroid".

While the emphasis on inflammation dominated research and treatment plans, it was easy to overlook other demonstrable means of inducing bronchospasm. Exercise-induced asthma and voluntary hyperventilation without exercise can individuals ${ }^{5}$ with relatively little inflammation.

However, despite the armamentarium of effective drugs, not all patients with asthma are fully controlled. Surveys have both induce bronchospasm in sensitive shown that, despite treatment, a significant proportion of patients have continuing symptoms ${ }^{6}$ such as continuing to wake several nights per week. Every clinician is aware that increasing doses of inhaled treatments is not effective in all patients. It is common to blame patient factors such as emotional overlays, poor compliance or poor inhaler technique for the drug failures. But it is also worth remembering that many patients with asthma exhibit a degree of hyperventilation with hypocapnia in the early phases of acute asthma.

Patients with asthma are relatively frequent users of alternative therapies, often without telling their physicians. ${ }^{8}$ The old medical adage advises "listen to the patient"; perhaps they are telling us something by their pursuit of buteyko, hypnosis, yoga or breathing exercises. All these share a common theme of attention to the breathing pattern. A Cochrane review concluded that, while there are insufficient data for firm conclusions about efficacy, there is enough evidence to justify further trials. ${ }^{9}$

The study by Holloway and West ${ }^{1}$ is interesting, but first one must acknowledge the negatives. It was an open study, it accepted the GP diagnosis without further proof and it relied on patient volunteers (who may not necessarily be representative). Indeed, at randomisation, the "active" group had more symptoms at the outset (ie, more room for improvement). And the same person delivered the treatments and recorded the outcomes. It cannot therefore be considered definitive. But, on the positive side, the study accepted most of the volunteers and is probably a more typical asthma primary care population than is included in many asthma drug trials. Randomised controlled trials have tended to categorise lung function and symptoms in great detail to avoid "contamination" both from patients without asthma and from patients with asthma who could not benefit. The non-selective intake to this study could potentially have diluted the ability to achieve measurable effects. This cohort of patients from general practice felt and functioned better. This is an outcome measure increasingly used in studies of chronic illness and the effects were present at 12 months, long after any placebo effect ought to have worn off.

Holloway and West describe an SGRQ benefit of 7 points compared with the control group. This is of similar magnitude to the randomised trials that led to long-acting $\beta$ agonists being adopted enthusiastically for the treatment of chronic obstructive pulmonary disease (eg, 6.7 points on the SGRQ). ${ }^{10}$ The changes reported using other measures 
of quality of life in asthma are also of a similar order. ${ }^{11}$ Such changes are therefore likely to be relevant to patients but cannot be ascribed to airway bronchodilation since $\mathrm{FEV}_{1}$ was unchanged. This then begs the questions as to what is being changed that makes patients feel better? Indeed, what was the abnormality that made these patients symptomatic in the first place? Is it part of the asthma or is it a concurrent problem?

Several discrete aspects need to be examined. The science around breathing control in the ambulant state remains quite limited. Chronic hyperventilation states are recognised, but the balance between psychic and somatic contributions to the overall state remains unclear. $^{12}{ }^{13}$ Some work does point towards respiratory physiological abnormalities, ${ }^{14}$ but there are few definitive answers. Next there is the role of breathing control and asthma. Asthma is hard to define and is multifactorial. Separating potential breathing control abnormalities from the airway effects of allergy, irritants, inflammation and exercise is challenging. And the questions continue as to whether it was the full Papworth technique or one component of it that produced the change.

However, regardless of whether the answers lie in a new physiological explanation or in an understanding of psychological reactions to the presence of a disease, the finding that something has made patients feel better means we cannot ignore this challenge. Five hours of therapist time for benefits that are apparent at 12 months compares well with the cost of a long-acting $\beta$ agonist for 1 year.

But these are difficult studies to deliver; adequate placebo control is difficult to contrive and they are time consuming to complete. It is an area that has little appeal for pharmaceutical or commercial support and it is hard to set out research protocols that will appeal to grant givers. Without financial support, the hurdles imposed by research governance, ethics committees and NHS pressures to work to targets mean that further studies may not be forthcoming. These workers are to be congratulated on completing this study without financial support.

Holloway and West's paper is not definitive and is insufficient to change management recommendations on its own. But the evidence that symptoms are not fully controlled by pharmacotherapy and the fact that many patients vote with their feet (and wallets) to seek outand apparently benefit from-alternative therapies should stimulate more studies. Maybe the time has come to tackle these symptoms from a different angle and to understand how psychology and the forgotten science of physiology may be as important to people with asthma as is the understanding of allergy and inflammation.

\section{Thorax 2007;62:1033-1034.}

doi: $10.1136 /$ thx.2007.084707

Correspondence to: Professor Mike G Pearson, Clinical Sciences Centre, University Hospital

Aintree, Longmoor Lane, Liverpool L9 7AL, UK; michael.pearson@liverpool.ac.uk
Competing interests: None.

\section{REFERENCES}

1 Holloway EA, West RJ. Integrated breathing and relaxation training (the Papworth method) for adults with asthma in primary care: a randomised controlled trial. Thorax 2007;62:1039-42.

2 Folgering $\mathbf{H}$. The hyperventilation syndrome. In: Altose MD, Kawakami Y, eds. Control of breathing in health and disease. New York, Basel: Marcel Dekker, 1999:633-60.

3 Howell JBL. Behavioural breathlessness. Thorax 1990:45:287-92.

4 Lum LC. The syndrome of habitual chronic hyperventilation. Recent Adv Psychosom Med 1976;3:196-230.

5 Sterk PJ, Fabbri LM, Quanjer PJ, et al. Airway responsiveness: standardised challenge testing with pharmacological, physicial and sensitising stimuli in adults. Eur Respir J, 1994:6(Suppl 16), 53-83.

6 European Community Respiratory Health Survey. Variations in the prevalence of respiratory symptoms, self-reported asthma attacks, and use of asthma medication in the European Community Respiratory Health Survey (ECRHS). Eur Respir J 1996;9:687-95.

7 Demeter SL, Lyons HA. Hyperventilation syndromes and asthma. Am J Med 1986;81:989-94.

8 Ernst E. Complementary therapies for asthma: what patients use. J Asthma 1998;35:667-71.

9 Holloway E, Ram F. Breathing exercises for asthma. In: The Cochrane Library, Issue 1. Oxford: Update Publications, 2004.

10 Jones PW, Bosch TK Quality of life changes in COPD patients treated with salmeterol. Am J Respir Crit Care Med 1997; 155:1283-9.

11 Rutten-van Molken MP, Custers F, van Doorslaer EK, et al. Comparison of performance of four instruments in evaluating the effects of salmeterol on asthma quality of life. Eur Respir J 1995;8:888-98.

12 Garder W. Orthostatic increase of respiratory gas volumes in hyperventilation syndromes. Thorax 2000;55:257-9.

13 Thomas M. Hyperventilation syndrome. Thorax 2005; $55: 884$

14 Jack S, Rossiter HB, Pearson MG, et al. Ventilatory responses to inhaled carbon dioxide, hypoxia and exercise in subjects with idiopathic hyperventilation Am J Respir Crit Care Med 2004;170: 1 18-25.

\section{What do non-eosinophilic asthma and airway remodelling tell us about persistent asthma?}

\section{Peter G Gibson}

\section{There is still much more to learn about the pathogenesis and treatment of asthma}

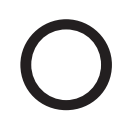
ver a decade of careful clinicopathological investigation has characterised the allergen-triggered Th2 response in asthma that leads to eosinophilic airway inflammation. This research has directed drug discovery programmes and we now have effective treatment for most steps in the eosinophilic asthma pathway. This list includes interventions that act at discrete levels such as allergen avoidance, allergen immunotherapy, anti-IgE antibodies, anti-interleukin-5 monoclonal antibodies and leucotriene receptor antagonists, together with corticosteroids that act on multiple levels in the pathway. Despite this significant success in therapeutic discovery, asthma persists. There must be something more to the pathogenesis of asthma. What could it be?

Airway remodelling and non-eosinophilic asthma (NEA) are both topical answers to this question. To date these have been pursued as distinct entities, but the paper by Berry and colleagues ${ }^{1}$ published in this issue of Thorax (see $\boldsymbol{p}$ 1043) addresses both issues and allows consideration of the interaction and overlap between airway remodelling and inflammatory subtype in asthma.

NEA refers to an asthma subtype where patients exhibit asthma symptoms and abnormal airway physiology (airway hyperresponsiveness (AHR), variable airflow obstruction) in the absence of a significant airway eosinophilia. ${ }^{2}$ Its importance arises because NEA is common, it seems to have a different 